power of the temperature, he considered a continuous arc impossible with our present refractory materials. There was further discussion by Messrs. Pike, C. Hering and Wahl.

Prof. L. F. Rondinella read a paper on "An Experimental Analogue for Direction of Induced Currents," which was discussed and referred for publication.

Prof. H. S. Hering gave the result of continued experiment on "The Effect of Atmospheric Pressure on Batteries," which seemed to show that the E. M. F. of standard cells did not become constant until about two hours after setting up. This result elıcited considerable discussion.

Upon motion of Mr. C. Hering, the treasurer was instructed to purchase a letter-box for the reception of queries on electrical subjects, which would be periodically submitted to the Section.

The meeting then adjourned.

L. F. Rondinella, Secretary.

\title{
AN EXPERIMENTAL ANALOGUE FOR DIRECTION OF INDUCED CURRENTS.
}

By L. F. Rondinella, M.E.

Prof, of Drawing, Central Manual Training School.

[Read at the stated meeting of the Electrical Section, April 7, I89r.]

The striking analogies which exist between current electricity and magnetism, form an important aid in acquiring a clear understanding of the fundamental principles of modern electrical science. For example, an electric current is assumed to pass through a generator from its soth to its north pole, and from thence through an external circuit back to the south pole again. So, also, are magnetic "currents," or lines of force assumed to pass through and outside of a magnet.

In an induced electric current lines of force are made to flow around a conductor in a right-handed or clockwise direction when the observer is looking along the conductor in the direction of the current, or toward the south pole of the generator. Similarly, in an induced- or electro-magnet, an electric current is made to flow around the core of the magnet right-handed when the observer is looking in the direction of the lines of force in the core, or toward the south pole of the magnet.

The directions of lines of force are usually discovered by 
the effect produced upon a small permanent magnet or magnetic needle, which will always come to rest with it, north pole pointing in the direction in which they flow through it. Then since the direction of a current depends upon the direction in which the lines of force flow round it, the former also is discovered by noticing the way in which the needle is deflected by the latter.

As there are two directions in which the current may flow through the conductor, and two positions in which the needle may be placed relative to it (above or below), it has been found necessary to use some memoria technica to quickly

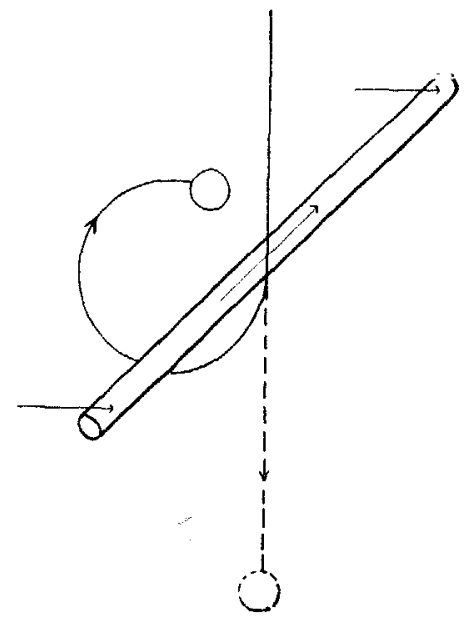

FIG. 1.

determine the direction of the current, from the way in which it deflects a needle; and among several devices that have been proposed, the following are most used, although they all present certain difficulties.

Ampère's rule is to imagine an observer swimming with the current in a conductor, with his face always toward the north pole of the needle, which will then be deflected toward his left hand. The disadvantages of this rule are the diffictilty of imagining the direction of the observer's left hand when he is placed in tuntsual positions, and the fact that it was intended for determining the deflection of the needle from the direction of the current which it pre- 
supposes to be known, and it must therefore be used inversely for our purpose.

Another device is to remember the fact that when a current flows from South to North Over a needle, its north pole points West, by noticing that the direction initials spell the word SNOW; and similarly. a third rule impresses the statement that when a current flows from North Over a needle to South its north pole point East, by spelling the word NOSE with the direction initials. A vital point that seems to be most troublesome to remember in these, is whether the current is over the needle or vice-versa, and the difficulty of always fincling a part of the conductor running north and south interferes with their direct application.

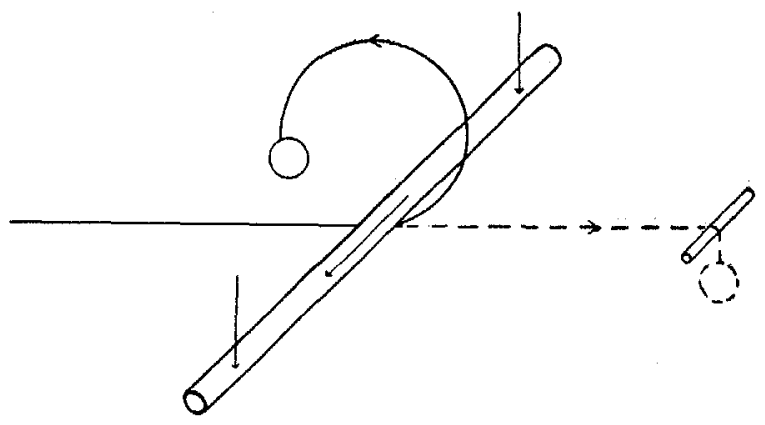

FIg. 2.

None of these devices can be used to predict the direction of the current that will be induced in a conductor when it is moved in a certain direction through a known magnetic field, and for this purpose the only one familiar to the writer is to use the right hand with $(a)$ the thumb, $(b)$ the first and $(c)$ the second finger pointed outward in the three rectangular directions to represent respectively $(a)$ the direction in which the conductor is moved, $(b)$ the direction of the lines of force, and $(c)$ the direction of the induced current. The trouble in applying this is to remember which hand to use and what each finger represents.

As an addition to these, and as an analogy that is not liable to confusion, the following experiment is suggested: If a small weight be suspended at the end of a piece of string (Fig. I), the force of the weight acts downward 
in the direction of the string, and the latter may be used to represent a "line of force" acting vertically downward. If then a horizontal rod, which may be used to represent a "conductor," be moved from left to right so as to strike sharply against the string a short distance above the weight, the latter with its "line of force" will revolve around the rod or "conductor" right-handed-a correct analogy to the phenomenon of electric induction. To represent lines of force acting horizontally in either direction, or vertically upward, the string may be held opposite or below a rod over which it is passed (Fig. 2), the force of the weight on its end acting along it in the proper direction. The rod may then be moved as desired, and the resulting revolution of the weight and string will remain a correct analogy for electric induction under similar circumstances. Then knowing the direction of the lines of force around the conductor in any case, the direction of the induced current flowing through it is readily determined by remembering that the observer is looking in the direction of the current when the lines of force flow right-handed around the conductor.

\section{A NEW FORM OF STANDARD CELL.}

By Carl Hering.

[Read at the stated meeting of the Electrical Section, held Feb. 24, 889. .]

The standard cells used at present may be said to be limited to the Clark cell and the Daniell cell. Each one of thesc has its specific advantages and disadvantages. The Clark cell is always ready for use, but is difficult to make, is affected by temperature, and has the important disadvantage that if by accident it is short-circuited it polarizes so rapidly and so much, that it is of nouse at all until it has been allowed to rest and recuperate for some time. Such accidental short circuits are not unlikely to occur, and they may furthermore occur, by the crossing of two wires, for instance, without the knowledge of the operator, thus making it possible that the 